Journal of Cognitive Neuroscience 2009; 21(9): 1834-1841

\title{
Learning to become an expert: reinforcement learning and the acqui- sition of perceptual expertise
}

\author{
Olav E. Krigolson, ${ }^{1,2}$ Lara J. Pierce, ${ }^{1}$ Clay B. Holroyd, ${ }^{1}$ James W. Tanaka ${ }^{1}$ \\ ${ }^{1}$ University of Victoria, ${ }^{2}$ University of British Columbia, Vancouver, CANADA \\ Correspondence : James W. Tanaka, E-mail: jtanaka@uvic.ca, Tel: +1250 721-7541
}

\section{Shruti Baijal}

Centre of Behavioural and Cognitive Sciences, University of Allahabad, Allahabad, U.P., INDIA

\section{Background}

Acquisition of perceptual skills is of vital importance as it enables identification of objects in the environment. With training and experience, it is possible to get specialized at recognizing a particular category of objects. The research on perceptual expertise has mostly contrasted performances and brain areas between experts and novices. The differences in perceptual capabilities between the experts and the novices rely on learning of implicit rules for categorization of objects ${ }^{1}$ and maintaining the domain-specific information in the working memory. ${ }^{2}$ There is evidence that acquisition of expertise is associated with activations in ventral visual stream, especially in the fusiform gyrus, the lateral occipital cortex ${ }^{3}$ and inferior temporal cortex. ${ }^{4}$

\section{Study Design}

Recently, Krigolson and his colleagues elegantly demonstrated the time course of brain activity underlying the development of perceptual expertise in novices ${ }^{5}$. They used eventrelated potential (ERP) measures in a categorization task. The human observers were required to learn to discriminate between two families of computer generated, two-dimensional polygons (learnable blobs) based on trial and error. The control condition used a third category of stimuli (morph blobs) that could not be classified as belonging to either family as they shared features with both groups. These blobs were presented in random order with the family names and the participants indicated whether they matched or not. Positive or negative feedback was provided after each response. Since the morph blobs did not belong to any particular category, the feedback for them was either positive or negative with a $50 \%$ probability. On the basis of the overall accuracy scores of the participants, they were divided into two groups - high learners (with high accuracy scores) and low learners (with low accuracy scores). The trials from the beginning and end portions of the experiment were used to compare the effects of trial and error based reinforcement learning in high and low performers. The performance of the high learners improved with practice of the learnable blobs only. However, the low learners did not show any improvement.

In terms of brain activity, three ERP components were observed - N250 elicited over occipitoparietal region (related to recognition of objects of expertise) along with response ERN (sensitive to correctness of responses) and feedback ERN (sensitive to processing of feedback) elicited over the frontalcentral region. Only the high learners learned to identify the learnable blobs that resulted in increase in the amplitudes of N250. Most importantly, with more training, the high learners developed the ability to evaluate the correctness of their responses while being less dependent on the external feedback. This was reflected as increase in amplitude of response ERN that preceded the enhancement of N250. In addition, there was a corresponding decrease in feedback ERN. The results suggested that the improvement in the categorization task was preceded by enhancement in the ability to evaluate the correctness of one's responses which reduced the dependence on any external feedback. Interestingly, no such effect was observed for the morph blobs.

\section{Implications}

The study clearly shows that the development of the ability to make object classifications is dependent upon feedbackrelated reinforcement learning. This begins with feedback signaling from the medial frontal areas or the neural generators of feedback $E R N^{5}$ to the early sensory areas for an initial evaluation of responses based on external feedback. The observers who successfully utilize this feedback are able to develop implicit rules for the categorization task. As the training progresses, these observers develop the ability to map the internally generated responses onto their early sensory percepts (as indicated by response ERN). This may eventually lead to better representations of the object categories in the early sensory areas (as indexed by N250) in the experts. Indeed, these findings delineated the precise processes underlying acquisition of perceptual expertise and led the authors to conclude that it marks a shift from the evaluation based on external feedback to the internal evaluation of one's responses.

Yet, it is to be noted that these findings are also associated with individual differences in terms of high and low performance. The differences in performance between individuals may be due to several reasons. Therefore, it is, perhaps, difficult to pinpoint a single cognitive process accountable for the individual differences in the ability to learn object-categorization. One possibility is that the individuals may differ based on their working memory performance. This opens interesting questions such as whether the differences between the high and low performers 
were due to the ability of high performers to efficiently encode and maintain information about expert domain objects.

Future work directed towards neurophysiological measurements of working memory capacity and its effect on the development of perceptual expertise are needed to better understand their relationship.

doi : 10.5214/ans.0972.7531.1118307

\section{References}

1. Ashby FG and Brien JB. Category learning and multiple memory systems. Trends Cog Sci 2005; 9: 83-89.
2. Chase WG and Simon HA. The mind's eye in chess. In: Chase WG Ed. Visual information processing. New York: Academic Press 1973; 215-281.

3. Gauthier I, Skudlarski P, Gore JC, et al. Expertise for cars and birds recruits brain areas involved in face recognition. Nat Neurosci 2000; 3: 191-197.

4. Kobatake E, Wang G and Tanaka K. Effects of shape-discrimination training on the selectivity of inferotemporal cells in adult monkeys. J Neurophysiol 1998; 80: 324-330.

5. Zaki SR, Nosofsky RM, Jessup NM, et al. Categorization and recognition performance of a memory-impaired group: Evidence for single-system models. J Int Neuropsychol Soc 2003; 9: 394-406. 